\title{
Movimientos de órganos y del paciente
}

\author{
L. E. Schiappacasse*, H. Marsiglia**
}

El movimiento de los órganos dentro del cuerpo puede ser clasificado globalmente como un movimiento aleatorio $\mathrm{y} / \mathrm{o}$ cíclico. Por ejemplo, se dice que la mayoría de los órganos en la pelvis se mueven aleatoriamente entre las fracciones de radiación y que los órganos abdominales se mueven cíclicamente debido a la respiración. Estos dos tipos de movimiento deben ser definidos en forma separada.

El desplazamiento temporal de la próstata es definido de manera similar a la desviación media en la posición de tratamiento (MTPD). La posición del órgano a partir de reparos óseos es definida en la simulación, y luego la desviación interfracción, a partir de esta posición, es medida durante el tratamiento, pudiendo objetivarse el desplazamiento de los órganos. De manera alternativa, las variaciones intrafracción pueden ser medidas utilizando imágenes dinámicas. Todos los desplazamientos de los órganos de cada paciente son combinados para formar un conjunto de datos a partir del cual es calculado el desplazamiento medio del órgano $\left(\mathrm{MOD}_{\text {organ }}\right)$, con su correspondiente desviación estándar. La desviación estándar del MOD es discutida de manera más extensa en la literatura que el MOD. Esto, porque el rango de los movimientos ha sido generalmente considerado de mayor importancia que la posición media del órgano. Sin embargo, el conocimiento en los últimos años del error sistemático que se encuentra al comparar las imágenes del scanner de planificación y la posición media del órgano durante el tratamiento fraccionado ha despertado creciente interés en el estudio de los errores sistemáticos en el movimiento de los órganos (van Herk et al, 1995).

Para aquellos órganos que se mueven predominantemente debido a las fuerzas respiratorias, por ejemplo los órganos abdominales, la mayoría de los autores miden el movimiento total del órgano. El movimiento total del órgano es definido como el cambio total en la posición del órgano desde la inspiración a la exhalación. De manera alternativa, para las técnicas "gated" que pueden incluir bloqueo de la respiración, la reproducibilidad de la posición del órgano en el mo-

* Instituto Oncológico, Viña del Mar, Chile

** Institut Gustave Roussy, Villejuif, Francia mento del bloqueo respiratorio o en una parte en particular del ciclo (por ejemplo, en la inspiración) es requerida.

\section{Magnitudes en el movimiento de los órganos}

El Internal Planning Target Volume (IPTV) describe un volumen que contiene todos los movimientos del CTV tomando como puntos de referencia la anatomía ósea. El CTV puede ser desplazado entre fracciones (variación interfracción) o durante la fracción (variación intrafracción). Las variaciones interfracción ocurren debido a diversos factores que incluyen, por ejemplo en los tratamientos de próstata, el hecho de que el paciente puede orinar antes de algunas fracciones, o puede no acostarse en la mesa de tratamiento de la misma manera para cada fracción. Las variaciones intrafracción pueden ocurrir debido a factores diversos que incluyen la respiración, los procesos digestivos o el latido del corazón o pulso de las arterias. Obviamente, la localización del tumor, la estructura y/o función de los órganos vecinos y el procedimiento de puesta en posición del paciente, tienen todos una influencia importante en los movimientos, su magnitud y el tamaño del IPTV necesario para incluir las incertidumbres. La literatura sugiere que muchas instituciones están comenzando a utilizar los valores de la media y la desviación estándar para la posición de órganos interfracción y las incertidumbres de posicionamiento para prescribir un margen "global", y no dos márgenes separados (interno y de posicionamiento), como lo sugiere el ICRU-62.

En la literatura, los órganos pelvianos (particularmente la próstata) y abdominales son los más estudiados. Uno de los desafíos al comparar la literatura publicada es la variación en las técnicas de medición utilizadas entre las diferentes instituciones.

\section{Movimientos de la próstata}

Las mediciones en la posición de la próstata han demostrado el movimiento del órgano debido a factores que incluyen el llene parcial del recto y la vejiga (variaciones interfracción) y también debido a la respiración (variaciones intrafracción). Diversos estudios han medido los movimientos de la próstata debidos específicamente a cada uno de estos factores y también excluyendo estos factores. Las variaciones 


\section{E. Schiappacasse y H. Marsiglia}

interfracción pueden ser grandes en algunas circunstancias. Sin embargo, la comprensión del por qué la próstata se mueve y cómo factores como un volumen rectal constante afectan la posición de la próstata y pueden ser de gran importancia para afinar la posición del campo de tratamiento.

La variación interfracción es causada predominantemente por diferencias en los volúmenes rectal y vesical en cada fracción (Melian et al 1997, Beard et al 1993). Recientemente, el movimiento de la próstata inducido por la respiración en pacientes que fueron posicionados en prono ha sido sugerido como una causa no trivial de movimientos (Malone et al 2000).

Las mediciones del movimiento prostático, obviando los volúmenes rectal y vesical, proveen valores similares de $M O D_{\text {prostate }}$ y $\sum_{O M}$ (desviación estándar del $M O D_{\text {prostate }}$ ).

Los valores $M O D_{\text {prostate }}$ presentados en la literatura tienen un rango de hasta $6 \mathrm{~mm}$, con _om que varía en el rango de $1.3 \mathrm{~mm}$ a $4.5 \mathrm{~mm}$. Los valores típicos (MODAS prostate ) se obtienen de las medidas $M O D_{\text {prostate, }}$ utilizando el centro de la masa prostática o marcadores individuales, con inmovilización o no del paciente durante el tratamiento.

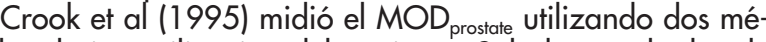
todos de inmovilización del paciente. Sólo los resultados de uno de los métodos (en que se medía el cambio de posición del centro de la masa de tres semillas de oro) fueron consistentes con las mediciones anteriormente practicadas. Los resultados de otro estudio demostraron que la próstata cambia su forma entre diferentes mediciones.

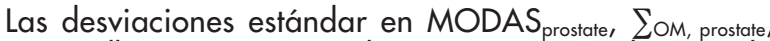
para aquellos pacientes tratados con un sistema de inmovilización son típicamente reportadas como similares a aquellas medidas sin el uso de uno de estos sistemas, aunque diversas instituciones han publicado mejores resultados utilizando sistemas de inmovilización.

La parte superior de la próstata y las vesículas seminales tienden a moverse más libremente en el sentido antero-posterior que el resto de la próstata (de un estudio con pacientes inmovilizados, Melian et al 1997). Los métodos para medir la posición de la próstata desde el centro de su masa asumen que la próstata no cambia su forma, pero se ha medido la deformación de la próstata (con consecuencias dosimétricas) debido a efectos fisiológicos (Forman et al 1993) como el llene rectal o vesical. Los métodos que usan marcadores o miden los cambios de posición de los bordes prostáticos pueden entregar una medición más representativa del $M O D_{\text {prostate }}$.

\section{Movimientos abdominales}

Mientras hipotéticamente el movimiento prostático es producido por la fisiología del paciente, el movimiento abdominal está habitualmente causado por la respiración y es en general cíclico. Durante los últimos 20 años, los métodos para cuantificar y manejar la respiración del paciente y el movimiento correspondiente de los órganos abdominales han progresado de la mano de los avances tecnológicos. El movimiento de los órganos dentro de la cavidad abdominal debido a la respiración ha sido cuantificado utilizando la tomografía axial computarizada (TAC) (Balter et al 1996), la resonancia nuclear magnética (RNM) (Morland et al 1994 Swartz et al 1994) y la ultrasonografía (US) (Oppelaar 1998, Davies et al 1994).

\section{Generalidades}

Las mediciones del $M O D_{\text {abdominal }}$ bajo las mismas condiciones clínicas (por ejemplo, frecuencia respiratoria), del mismo órgano, en una dirección dada y presentadas en la misma forma no se encuentran en la literatura. Por esta razón, los valores típicos del $M O D_{\text {abdominal }}$ no pueden ser deducidos.

Utilizando un TAC ultra rápido durante la respiración normal, Ross et al (1990) midió rangos de hasta $22 \mathrm{~mm}$ en los movimientos abdominales totales en la dirección medial-lateral (con una media de $6.1 \mathrm{~mm}$ ) y de hasta $15 \mathrm{~mm}$ en la dirección antero-posterior (con una media de $1.9 \mathrm{~mm}$ ). Los movimientos cráneo-caudales fueron mayores cerca del diafragma, mientras los movimientos laterales fueron mayores cerca del corazón o la aorta. Casamassima (1994) midió movimientos de hasta $3 \mathrm{~cm}$ del riñón e hígado durante la respiración normal. Davies et al (1994) encontró que el movimiento abdominal era mayor para el diafragma, moderado para los riñones y el hígado, con movimientos del diafragma y del hígado predominantemente en la dirección superior-inferior. Los tumores del lóbulo superior y aquellos adheridos a la pared torácica mostraban movimientos mínimos.

Las dificultades en la comparación de resultados son la falta de información direccional medida para el movimiento, las diferentes frecuencias respiratorias de los pacientes/voluntarios en cada estudio, y los diversos objetivos de cada medición.

Como debe esperarse, un movimiento de órganos mayor fue reportado en cada sitio durante la respiración forzada que durante la respiración normal. Los movimientos de órganos han demostrado seguir patrones complejos cuando el paciente respira forzadamente. Sin embargo, la mayoría de los estudios considera que la respiración normal muestra amplitudes de movimiento reproducibles (con desviaciones estándar de 6 a $8 \mathrm{~mm}$ para los riñones, y de hasta $30 \mathrm{~mm}$ para otros órganos abdominales).

\section{Movimiento del riñón}

Schwartz et al (1994) midieron rangos de movimiento total del riñón de hasta $43 \mathrm{~mm}$ entre la inhalación y exhalación, con una reproducibilidad (desviación estándar del movimiento medio total) en la inhalación de entre $1.8 \mathrm{~mm}$ a $3 \mathrm{~mm}$ y de $2.8 \mathrm{~mm}$ a $3.6 \mathrm{~mm}$ para la exhalación, utilizando RNM ultra rápida. No se encontraron diferencias entre los movimientos del riñón izquierdo y derecho, como lo midió Balter et al (1996) o Suramo et al (1984) durante la respiración normal. Moerland et al (1984) encontraron que el rango de desplazamiento del riñón derecho era mayor que el del riñón izquierdo durante la respiración normal. Tanto Moerland et al (1984) como Ahmad et al (1997) encontraron mayor movimiento y variabilidad de los movimientos en el riñón izquierdo que en el riñón derecho durante la respiración forzada.

\section{Movimientos del páncreas}

Kivisaari et al (1982) midieron el movimiento total del páncreas y encontraron que era independiente de la condición del páncreas, además de ser más móvil en aquellas personas cuyo páncreas era considerado normal, o sufrían de 
pancreatitis, que en aquellos pacientes con carcinoma pancreático. Los páncreas con las primeras dos condiciones tendían a ser más móviles en la cola, siendo el cuerpo menos móvil. Aquellos páncreas afectados por carcinoma tendían a moverse más en el cuerpo y menos en su cola. Las mediciones del movimiento del páncreas normal de Suramo et al (1984) mostraron menores movimientos que el estudio de Kivisari et al (1982).

\section{Técnicas de bloqueo respiratorio}

Kuhns et al (1979) demostraron que el movimiento de los polos superiores renales era de $4.9 \pm 8.3 \mathrm{~mm}$ durante la inspiración y de $7.7 \pm 6.6 \mathrm{~mm}$ durante la expiración. Suramo et al (1984) encontraron un método, utilizando una barra horizontal (con la capacidad de moverse verticalmente) puesta sobre el tórax del paciente, para reducir el movimiento de los órganos. En particular, los movimientos de los riñones, hígado y páncreas fueron disminuidos de $4 \mathrm{~mm}$ a $1 \mathrm{~mm}, 9$ $\mathrm{mm}$ a $2 \mathrm{~mm}$, y $6 \mathrm{~mm}$ a $1 \mathrm{~mm}$, respectivamente.

\section{Conclusiones}

El considerar la inevitable incertidumbre durante la planificación y tratamiento con radioterapia reviste una importancia fundamental. En todos los sitios, la inclusión del conocimiento de las magnitudes y características de los errores debe ser considerada. Esto, debe combinarse con técnicas para controlar la magnitud de los errores. La mejor oportunidad para darse cuenta de estas incertidumbres es el proceso de planificación, al utilizar métodos que reflejen la naturaleza estadística de muchos de los errores.

Claramente, la predicción del movimiento potencial del órgano y la posición del paciente son la mejor opción para intentar corregir los potenciales errores sistemáticos de posición de los órganos entre la planificación y el tratamiento. 